PROCEEDINGS OF THE

AMERICAN MATHEMATICAL SOCIETY

Volume 136, Number 11, November 2008, Pages 4019-4025

S 0002-9939(08)09373-8

Article electronically published on June 4, 2008

\title{
ZERO SETS OF SMOOTH FUNCTIONS IN THE PFAFFIAN CLOSURE OF AN O-MINIMAL STRUCTURE
}

\author{
G. O. JONES
}

(Communicated by Julia Knight)

\begin{abstract}
I show that in an o-minimal structure on the real field, satisfying certain conditions, every closed definable set is the zero set of a smooth definable function. The conditions are shown to hold in the Pfaffian closure of a polynomially bounded o-minimal structure having smooth cell decomposition.
\end{abstract}

\section{INTRODUCTION}

Suppose that $X \subseteq \mathbb{R}^{n}$ is closed. Then by a theorem of Whitney, there is a smooth (i.e. $C^{\infty}$ ) function $f: \mathbb{R}^{n} \rightarrow \mathbb{R}$ vanishing precisely on $X$ such that $f$ is analytic off $X$. The question I shall address in this note is, if we suppose in addition that $X$ is definable in some o-minimal structure $\mathcal{R}$ on the real exponential field, can $f$ be taken to be definable in $\mathcal{R}$ ?

Progress on this question was first made by van den Dries and Miller (3]). Suppose that $\mathcal{R}$ is an o-minimal expansion of the real field and let $p \in \mathbb{N}$. Suppose that $X \subseteq \mathbb{R}^{n}$ is closed and definable. Then van den Dries and Miller show that there is a definable $C^{p}$ function $f: \mathbb{R}^{n} \rightarrow \mathbb{R}$ such that $f$ vanishes precisely on $X$.

To obtain a smooth function, the exponential function is necessary. To see this, first note that, by a theorem of Miller ([7]), if the exponential function is not definable, then the structure is polynomially bounded (that is, for every definable unary function $g$ there is some $m \in \mathbb{N}$ such that, for large $\left.t,|f(t)|<t^{m}\right)$. Now, Miller ([8) has also proved that in a polynomially bounded o-minimal structure, if a smooth definable function has a flat point (that is, a point at which the function, and its derivatives of all orders, vanish), then it vanishes locally around that point. In particular, in a polynomially bounded o-minimal structure, the interval $[0,1]$ is not the zero set of a definable smooth function.

Now suppose again that the exponential function is definable in $\mathcal{R}$ (in which case, the structure is said to be exponential). Most of the proof in [3] goes through in the smooth case, but the finiteness of $p$ is used to preserve definability. I shall show that this is not necessary, provided we know that the structure has smooth cell decomposition and we have certain bounds on the derivatives (of all orders) of definable smooth functions. I shall assume that the reader is familiar with standard results on o-minimality; see van den Dries' book 2]. To state the precise result, I need to introduce some further terminology.

Received by the editors July 23, 2007, and, in revised form, October 5, 2007.

2000 Mathematics Subject Classification. Primary 03C64; Secondary 58A35.

The author is supported by NSERC.

(C)2008 American Mathematical Society
Reverts to public domain 28 years from publication 4019 
Definition 1.1. Let $f: U \rightarrow \mathbb{R}$ be a smooth definable function on an open set $U \subseteq$ $\mathbb{R}^{n}$. We say that $f$ has controlled derivatives if there exists a definable continuous function $\omega: U \rightarrow \mathbb{R}$, real numbers $C_{1}, C_{2}, \ldots$ and natural numbers $E_{1}, E_{2}, \ldots$ such that

$$
\left|D^{\alpha} f(x)\right| \leq C_{|\alpha|} \omega(x)^{E_{|\alpha|}}
$$

for all $x \in U$ and $\alpha \in \mathbb{N}^{n}$. Here and throughout, we write $D^{\alpha}$ for the differential operator $\frac{\alpha^{|\alpha|}}{\partial x_{1}^{\alpha_{1}} \ldots \partial x_{n}^{\alpha_{n}}}$ and $|\alpha|=\alpha_{1}+\cdots+\alpha_{n}$. We say that a structure $\mathcal{R}$ on the real field has piecewise controlled derivatives if, for every definable smooth function $f: U \rightarrow \mathbb{R}$, there are definable open sets $U_{1}, \ldots, U_{k} \subseteq U$ such that $\operatorname{dim} U \backslash \bigcup U_{i}<n$ and, for each $i,\left.f\right|_{U_{i}}$ has controlled derivatives.

Theorem 1.2. Suppose that $\mathcal{R}$ is an exponential o-minimal structure having smooth cell decomposition and piecewise controlled derivatives. Then every closed definable set $X \subseteq R^{n}$ is the zero set of a definable smooth function $f: \mathbb{R}^{n} \rightarrow \mathbb{R}$.

The obvious question in response to this is: what structures have piecewise controlled derivatives? In 4, Wilkie and I showed that locally polynomially bounded structures satisfying an additional condition have piecewise controlled derivatives. See our paper for precise definitions and results. Lion and Speissegger have shown ([6]) that if $\mathcal{R}$ has smooth cell decomposition, then so does $\mathcal{P}(\mathcal{R})$, the Pfaffian closure of $\mathcal{R}$ (the o-minimality of $\mathcal{P}(\mathcal{R})$ is due to Speissegger [10]). I use their methods to prove the following.

Theorem 1.3. Suppose $\mathcal{R}$ has smooth cell decomposition and piecewise controlled derivatives. Then $\mathcal{P}(\mathcal{R})$ has piecewise controlled derivatives.

Now, any polynomially bounded structure with smooth cell decomposition has piecewise controlled derivatives. Lion, Miller and Speissegger proved ([5]) that the Pfaffian closure of a polynomially bounded structure is exponentially bounded. Combined with the results above, this easily implies the following.

Corollary 1.4. Suppose $\mathcal{R}$ is a polynomially bounded o-minimal structure with smooth cell decomposition. Then every exponential reduct of $\mathcal{P}(\mathcal{R})$ having smooth cell decomposition has piecewise controlled derivatives and each closed definable set is the zero set of a smooth definable function.

At the present time, all known exponential structures (see Speissegger's survey 9]) satisfy the hypothesis of the corollary. Despite these examples, Theorem 1.2 is far from optimal. It requires two rather strong properties (in addition to ominimality) of the structure $\mathcal{R}$. To make any progress on removing these assumptions, new ideas are needed. In addition, the question of whether a function $f$ can be found which is also analytic off $X$ remains open. Finally, I thank the referee for pointing out that the following question is also open. Suppose that $X \subseteq \mathbb{R}^{n}$ is any closed set (not necessarily definable in an o-minimal structure). Can a function $f$ be found, which, in addition to satisfying the conclusion of Whitney's result, is definable in the structure $(\mathbb{R},+, \cdot, \exp , X)$ ?

Before beginning the proofs, we fix some notation. For a set $X \subseteq \mathbb{R}^{n}$, we let $\operatorname{bd}(X)$ be the boundary of $X$. Given a function $f$, we let $Z(f)=\{x \in \operatorname{dom} f$ : $f(x)=0\}$. For $r \in \mathbb{R}$, we let $\mathbb{R}_{\geq r}=\{t \in \mathbb{R}: t \geq r\}$. If $N \geq n$, we let $\pi_{n}: \mathbb{R}^{N} \rightarrow \mathbb{R}^{n}$ be projection onto the first $n$ coordinates. 


\section{THE ZERO SET PROPERTY}

In this section we prove Theorem 1.2, So, let $\mathcal{R}$ be an exponential o-minimal expansion of the real field and assume that $\mathcal{R}$ has smooth cell decomposition and piecewise controlled derivatives. With these assumptions, we only need to make a small adjustment to the proof in 3 . First, note that the proofs of C.5 to C. 8 of 3] go through with $p=\infty$. We need the following version of [3, C.9], in which $\Phi$ denotes the collection of increasing bijections $\phi: \mathbb{R} \rightarrow \mathbb{R}$ which are smooth, definable and flat at 0 .

Proposition 2.1. Let $f, g: \mathbb{R}^{n} \rightarrow \mathbb{R}$ be continuous definable functions with $Z(f) \subseteq$ $Z(g)$. Suppose that on $\mathbb{R}^{n} \backslash Z(g)$, the functions $f$ and $g$ are smooth and have controlled derivatives. Then there exists $\phi \in \Phi$ and a smooth definable function $h: \mathbb{R}^{n} \rightarrow \mathbb{R}$, both with controlled derivatives, such that

$$
\phi \circ g=h \cdot f \text {. }
$$

Proof. Let $U=\mathbb{R}^{n} \backslash Z(g)$. Since $f$ and $g$ have controlled derivatives on $U$, we can find a continuous definable function $\omega: U \rightarrow \mathbb{R}_{\geq 0}$ such that (1.1) holds for both $f$ and $g$. Now, by the $p=\infty$ version of [3, C.8], there exists $\psi \in \Phi$ such that for all $y \in \operatorname{bd}(U)$ we have

$$
\lim _{\substack{x \rightarrow y \\ x \in U}} \psi(g(x)) \frac{\omega(x)}{f(x)}=0 .
$$

Now suppose that $\theta: \mathbb{R} \rightarrow \mathbb{R}$ is any smooth definable function which is flat at 0 and non-zero for small $t$. An easy argument using (2.1) and the fact that $\theta$ is flat at 0 shows that for all $l, k \in \mathbb{N}$ and $y \in \operatorname{bd}(U)$, we have

$$
\lim _{\substack{x \rightarrow y \\ x \in U}} \theta(\psi(g(x))) \frac{\omega(x)^{l}}{f(x)^{k}}=0 .
$$

In particular, if $r \in \mathbb{N}$, then (2.2) holds for $\theta=\psi^{(r)}$.

Let $\phi=\psi \circ \psi$ and note that $\phi \in \Phi$. Define $h: \mathbb{R}^{n} \rightarrow \mathbb{R}$ by

$$
h(x)= \begin{cases}\frac{\phi(g(x))}{f(x)} & x \in U, \\ 0 & x \notin U .\end{cases}
$$

Let $\alpha \in N^{n}$. Using the chain rule and induction on $|\alpha|$, it is easy to see that $D^{\alpha} h$ is a sum of terms of the form

$$
\frac{\left(\psi^{(r)} \circ g\right)\left(\psi^{(s)} \circ \psi \circ g\right)\left(D^{\alpha_{1}} g\right)^{l_{1}} \cdots\left(D^{\alpha_{p}} g\right)^{l_{p}}\left(D^{\beta_{1}} f\right)^{k_{1}} \cdots\left(D^{\beta_{q}} f\right)^{k_{q}}}{f^{m}}
$$

for various $r, s, l_{i}, k_{j}, p, q, m \in \mathbb{N}$ and $\alpha_{i}, \beta_{j} \in \mathbb{N}^{n}$. Now, because (1.1) holds for both $f$ and $g$, a term of this form has absolute value bounded by

$$
C \cdot\left|\psi^{(r)} \circ g \| \psi^{(s)} \circ \psi \circ g\right| \frac{\omega^{l}}{|f|^{k}}
$$

for some $k, l \in \mathbb{N}$ and $C \in \mathbb{R}$. Since $\psi^{(s)}(g(x)) \rightarrow 0$ as $x \rightarrow y$ for $y \in \operatorname{bd}(U)$, it follows from (2.2) that each of the terms above approaches 0 as $x$ approaches $\operatorname{bd}(U)$. Hence for each $y \in \operatorname{bd}(U)$ we have

$$
\lim _{\substack{x \rightarrow y, x \in U}} D^{\alpha} h(x)=0,
$$

and so by monotonicity and l'Hôpital's rule, $h$ is smooth on $\mathbb{R}^{n}$. 
To see that $h$ has controlled derivatives, first note that since $\mathcal{R}$ is o-minimal and has piecewise controlled derivatives, any smooth unary definable function has controlled derivatives on its whole domain. So, we may choose a continuous definable function $\theta: \mathbb{R} \rightarrow \mathbb{R}_{\geq 0}$ and sequences $C_{1}, C_{2}, \ldots, E_{1}, E_{2}, \ldots$ such that

$$
\left|\psi^{(r)}(t)\right| \leq C_{r} \theta(t)^{E_{r}}
$$

for all $r \in \mathbb{N}$ and $t \in \mathbb{R}$. Since each of the functions $\psi^{(r)}$ is flat at 0 , we may choose $\theta$ such that $0<\theta(t)<|t|$ for small $t$. Hence by (2.2), the function $\omega_{1}: \mathbb{R}^{n} \rightarrow \mathbb{R}_{\geq 0}$ defined by

$$
\omega_{1}(x)= \begin{cases}\theta(\psi(g(x))) \frac{\omega(x)}{|f(x)|} & x \in U, \\ 0 & x \notin U\end{cases}
$$

is continuous. Now define $\omega_{2}(x): \mathbb{R}^{n} \rightarrow \mathbb{R}$ by $\omega_{2}(x)=\theta(g(x)) \cdot \omega_{1}(x)$. Then $\omega_{2}$ is continuous and definable, and because of the bounds in (2.4), we can find sequences $C_{1}^{\prime}, C_{2}^{\prime}, \ldots, E_{1}^{\prime}, E_{2}^{\prime}, \ldots$ such that

$$
\left|D^{\alpha} h(x)\right| \leq C_{|\alpha|}^{\prime} \omega_{2}(x)^{E_{\alpha}^{\prime}}
$$

for all $\alpha \in \mathbb{N}^{n}$ and $x \in \mathbb{R}^{n}$, and so $h$ has controlled derivatives.

We need some closure properties of functions with controlled derivatives, which follow easily from the chain rule.

Proposition 2.2. Let $U$ be a definable open subset of $\mathbb{R}^{n}$. The collection $\mathcal{C}_{c d}^{\infty}(U)$ of all smooth definable functions with controlled derivatives on $U$ is a ring. If $f=\left\langle f_{1}, \ldots, f_{n}\right\rangle: V \rightarrow U$ is a smooth definable map on a definable open subset $V$ of $\mathbb{R}^{m}$, with $f_{1}, \ldots, f_{n} \in \mathcal{C}_{c d}^{\infty}(V)$ and $g \in \mathcal{C}_{c d}^{\infty}(U)$, then

$$
g \circ f \in \mathcal{C}_{c d}^{\infty}(V) .
$$

Theorem 2.3. Suppose that $X \subseteq \mathbb{R}^{n}$ is a closed definable set. Then there exists $f \in \mathcal{C}_{c d}^{\infty}\left(\mathbb{R}^{n}\right)$ such that $X=Z(f)$.

Proof. Follow the proof of C.11 in [3] (in which there is a slight gap at the start of the third paragraph, use the maps $\tau_{n}$ from [3, §3] to reduce to the compact case). Refer to Proposition 2.1 in place of C.9 (and the obvious corollary to Proposition 2.1 in place of C.10). To see that the resulting function has controlled derivatives, use the fact that the unary smooth definable functions and the function $h$ provided by Proposition 2.1 have controlled derivatives, and use Proposition 2.2 .

\section{Controlling Derivatives in the Pfaffian Closure}

In this section we prove Theorem 1.3. For the moment, let $\mathcal{R}$ be any o-minimal expansion of the real field, and let $\mathcal{P}(\mathcal{R})$ denote the Pfaffian closure of $\mathcal{R}$. We use results from ([6]). First we recall some definitions and notation.

For $p \leq n$, we let $G_{n}^{p}$ be the Grassmannian of all $p$-dimensional vector subspaces of $\mathbb{R}^{n}$. Then $G_{n}^{p}$ is a non-singular real algebraic variety and hence a real analytic manifold. It has a natural analytic embedding in the space of all $n \times n$ matrices. See [1] for details. We identify $G_{n}^{p}$ with its image in the space of $n \times n$ matrices. Fix $k \in\{1,2, \ldots, \infty\}$ and let $M \subseteq \mathbb{R}^{n}$ be an embedded $C^{k}$-submanifold.

Definition 3.1. A p-distribution (of class $C^{k}$ ) on $M$ is a $C^{k}$ map $\mathcal{D}: M \rightarrow G_{n}^{p}$ such that $\mathcal{D}(x) \subseteq T_{x} M$ for all $x \in M$. Let $\mathcal{D}$ be a distribution on $M$. An immersed submanifold $X \subseteq M$ is called an integral manifold of $\mathcal{D}$ if $T_{x} X=\mathcal{D}(x)$ for all 
$x \in X$. Note that we do not assume that integral manifolds are maximal. A vector field $v$ on $M$ is tangent to $\mathcal{D}$ if $v(x) \in \mathcal{D}(x)$ for all $x \in M$. Let $\mathcal{V}^{1}(M, \mathcal{D})$ be the set of all $C^{1}$ vector fields on $M$ which are tangent to $\mathcal{D}$ and let

$$
I(\mathcal{D})=\left\{x \in M:[v, w](x) \in \mathcal{D}(x) \text { for all } v, w \in \mathcal{V}^{1}(M, \mathcal{D})\right\},
$$

where $[v, w]$ is the Lie bracket of $v$ and $w$. We say that $\mathcal{D}$ is integrable if $I(\mathcal{D})=M$.

We say that $M \subseteq \mathbb{R}^{n}$ is an $\mathcal{R}$-definable submanifold if $M$ is an embedded $C^{1}$ submanifold of $\mathbb{R}^{n}$ and an $\mathcal{R}$-definable set.

We need several results of Lion and Speissegger.

Theorem 3.2 (Corollary 5.2 in [6]). Suppose that $S \subseteq \mathbb{R}^{n}$ is definable in $\mathcal{P}(\mathcal{R})$. Then there are $C^{1}$ cells $S_{1}, \ldots, S_{k}$, definable in $\mathcal{P}(\mathcal{R})$ with $S=S_{1} \cup \cdots \cup S_{k}$ such that for each $S^{\prime}=S_{i}$ the following holds: there exist $N \geq n$, an $\mathcal{R}$-definable manifold $M \subseteq \mathbb{R}^{N}$, an integrable $C^{1} \mathcal{R}$-definable $p$-distribution $\mathcal{D}$ on $M$ where $p=\operatorname{dim} S^{\prime}$, and a $C^{1}$ cell $X \subseteq M$ definable in $\mathcal{P}(\mathcal{R})$ such that $X$ is an integral manifold of $\mathcal{D}$ and $S^{\prime}=\pi_{n}(X)$.

Theorem 3.3 (Corollary 5.4 in [6]). If $\mathcal{R}$ has smooth cell decomposition, then so does $\mathcal{P}(\mathcal{R})$.

We now assume that $\mathcal{R}$ has smooth cell decomposition.

Theorem 3.4. Suppose that $f: U \rightarrow \mathbb{R}$ is smooth and definable in $\mathcal{P}(\mathcal{R})$, where $U$ is an open set in $\mathbb{R}^{n}$. Then there are smooth cells $C_{1}, \ldots, C_{l} \subseteq U$, definable in $\mathcal{P}(\mathcal{R})$ such that $U=C_{1} \cup \cdots \cup C_{l}$ and for each $C=C_{i}$ the following holds: there exist $k \geq 0$, smooth functions $f_{1}, \ldots, f_{k}: C \rightarrow \mathbb{R}$ definable in $\mathcal{P}(\mathcal{R})$, and smooth $\mathcal{R}$-definable functions $P_{1}, \ldots, P_{k}: D \rightarrow \mathbb{R}$, where $D$ is a smooth cell containing $\Gamma\left(\left\langle\left. f\right|_{C}, f_{1}, \ldots, f_{k}\right\rangle\right)$, such that

$$
\frac{\partial f}{\partial x_{i}}(x)=P_{i}\left(x, f(x), f_{1}(x), \ldots, f_{k}(x)\right)
$$

for all $x \in C$ and each $i=1, \ldots, n$.

Proof. Apply Theorem 3.2 to the graph of $f$. Then it suffices to prove the result for the restriction of $f$ to each of the resulting cells. Hence we may suppose that we have $N \geq n+1$, an $\mathcal{R}$-definable manifold $M \subseteq \mathbb{R}^{N}$, an integrable $C^{1} \mathcal{R}$ definable $n$-distribution $\mathcal{D}$ on $M$ and a $\mathcal{P}(\mathcal{R})$-definable $C^{1} n$-cell $X \subseteq M$ such that $X$ is an integral manifold of $\mathcal{D}$ and $\pi_{n+1}(X)=\Gamma(f)$. It follows that there are $\mathcal{P}(\mathcal{R})$-definable functions $f_{1}, \ldots, f_{k}: U \rightarrow \mathbb{R}$, where $k=N-(n+1)$ such that $X=\Gamma\left(\left\langle f, f_{1}, \ldots, f_{k}\right\rangle\right)$. Using smooth cell decomposition in the structures $\mathcal{R}$ and $\mathcal{P}(\mathcal{R})$, we may suppose that all of the above data is smooth. In particular, we may assume that the projection map $\pi=\pi_{n}: \mathbb{R}^{N} \rightarrow \mathbb{R}^{n}$ restricts to a diffeomorphism of $X$ onto $U$.

For each $y \in X$, there is an induced isomorphism $\pi_{*}: T_{y} X \rightarrow T_{\pi(y)} U=T_{\pi(y)} \mathbb{R}^{n}$. Note that for $x \in U$ and $i=1, \ldots, n$, the image under $\pi_{*}$ of the tangent vector

$$
\left\langle 0, \ldots, 0,1,0, \ldots, 0, \frac{\partial f}{\partial x_{i}}(x), \frac{\partial f_{1}}{\partial x_{i}}(x), \ldots, \frac{\partial f_{k}}{\partial x_{i}}(x)\right\rangle
$$

is the standard basis vector $\langle 0, \ldots, 0,1,0, \ldots, 0\rangle$ in $\mathbb{R}^{n}$.

Let $Y$ be the set of points $y \in M$ such that there is a unique basis of $\mathcal{D}(y)$ which maps to the standard basis of $T_{\pi(y)} \mathbb{R}^{n}$ under $\pi_{*}$. Note that $Y$ is $\mathcal{R}$-definable. Since $X$ is an integral manifold of $\mathcal{D}$, we have $\mathcal{D}(y)=T_{y} X$ for each $y \in X$, so $X \subseteq Y$. 
Let $P$ be the $\mathcal{R}$-definable map which takes each $y \in Y$ to the corresponding basis of $\mathcal{D}(y)$. Now, if $x \in U$, then $P\left(x, f(x), f_{1}(x), \ldots, f_{k}(x)\right)$ is the basis

$$
\begin{array}{r}
\left\{\left\langle 1,0, \ldots, 0, \frac{\partial f}{\partial x_{1}}(x), \frac{\partial f_{1}}{\partial x_{1}}(x), \ldots, \frac{\partial f_{k}}{\partial x_{1}}(x)\right\rangle,\right. \\
\left.\ldots,\left\langle 0, \ldots, 0,1, \frac{\partial f}{\partial x_{n}}(x), \frac{\partial f_{1}}{\partial x_{n}}(x), \ldots, \frac{\partial f_{k}}{\partial x_{n}}(x)\right\rangle\right\} .
\end{array}
$$

Hence there are $\mathcal{R}$-definable functions $P_{i}$ such that

$$
\frac{\partial f}{\partial x_{i}}(x)=P_{i}\left(x, f(x), f_{1}(x), \ldots, f_{k}(x)\right)
$$

for all $x \in U$ and $i=1, \ldots, n$. The result now follows from smooth cell decomposition (in $\mathcal{R}$ ).

Proposition 3.5. Suppose that $\mathcal{R}$ has piecewise controlled derivatives. Let $f_{1}, \ldots$, $f_{k}: U \rightarrow \mathbb{R}$ be smooth $\mathcal{P}(\mathcal{R})$-definable functions on an open set $U \subseteq \mathbb{R}^{n}$ and $P_{1}, \ldots$, $P_{n}: D \rightarrow \mathbb{R}$ be smooth $\mathcal{R}$-definable functions on a smooth cell $D$ containing the graph of the map $\left\langle f_{1} \ldots, f_{k}\right\rangle$. Suppose that for all $x \in U$ and $i=1, \ldots, k$ we have

$$
\frac{\partial f_{1}}{\partial x_{i}}(x)=P_{i}\left(x, f_{1}(x), \ldots, f_{k}(x)\right) .
$$

Then there are $\mathcal{P}(\mathcal{R})$-definable open sets $U_{1}, \ldots, U_{r} \subseteq U$ such that $\operatorname{dim} U \backslash \bigcup U_{j}<n$, and, for each $j=1, \ldots, r$, the restriction of $f_{1}$ to $U_{j}$ has controlled derivatives.

Proof. This is an easy computation using the chain rule and the fact that $\mathcal{R}$ has piecewise controlled derivatives.

Combining the results of this section, we obtain the following.

Corollary 3.6. If $\mathcal{R}$ has piecewise controlled derivatives and smooth cell decomposition, then the same is true of $\mathcal{P}(\mathcal{R})$.

\section{ACKNOWLEDGMENTS}

The author thanks Chris Miller, Patrick Speissegger and Alex Wilkie for helpful discussions, and the referee for useful suggestions.

\section{REFERENCES}

[1] J. Bochnak, M. Coste, and M.-F. Roy, Real algebraic geometry, Ergebnisse der Mathematik und ihrer Grenzgebiete (3) [Results in Mathematics and Related Areas (3)], vol. 36, SpringerVerlag, Berlin, 1998. Translated from the 1987 French original, revised by the authors. MR:1659509 (2000a:14067)

[2] L. van den Dries, Tame topology and o-minimal structures, London Mathematical Society Lecture Note Series, vol. 248, Cambridge University Press, Cambridge, 1998. MR1633348 (99j:03001)

[3] L. van den Dries and C. Miller, Geometric categories and o-minimal structures, Duke Math. J. 84 (1996), no. 2, 497-540. MR.1404337 (97i:32008)

[4] G. O. Jones and A. J. Wilkie, Locally polynomially bounded structures, Bull. London Math. Soc. 40 (2008), 239-248.

[5] J.-M. Lion, C. Miller, and P. Speissegger, Differential equations over polynomially bounded o-minimal structures, Proc. Amer. Math. Soc. 131 (2003), no. 1, 175-183 (electronic). MR 1929037 (2003g:03064)

[6] J.-M. Lion and P. Speissegger, Analytic stratification in the Pfaffian closure of an o-minimal structure, Duke Math. J. 103 (2000), no. 2, 215-231. MR.1760626 (2001j:03076) 
[7] C. Miller, Exponentiation is hard to avoid, Proc. Amer. Math. Soc. 122 (1994), no. 1, 257259. MR.1195484 (94k:03042)

[8] _ Infinite differentiability in polynomially bounded o-minimal structures, Proc. Amer. Math. Soc. 123 (1995), no. 8, 2551-2555. MR1257118 (95j:03069)

[9] P. Speissegger, O-minimal expansions of the real field, Model theory and applications, Quad. Mat., vol. 11, Aracne, Rome, 2002, pp. 379-408. MR2159726 (2006m:03066)

[10] 189-211. MR1676876 (2000j:14093)

Department of Mathematics and Statistics, McMaster University, 1280 Main Street, West Hamilton, Ontario L8S 4K1, Canada

Current address: School of Mathematics, University of Manchester, Oxford Road, Manchester, M13 9PL, United Kingdom

E-mail address: gojones@math.mcmaster.ca 